\title{
PRÉ-CONCENTRAÇÃO DE CÁDMIO COM Saccharomyces cerevisiae E DETERMINAÇÃO EM ÁGUAS FLUVIAIS USANDO ESPECTROMETRIA DE EMISSÃO ÓPTICA COM PLASMA INDUTIVAMENTE ACOPLADO
}

\author{
Priscila Andreoli Biscaro e Amauri Antonio Menegário* \\ Centro de Estudos Ambientais, Universidade Estadual Paulista, Av. 24-A, 1515, 13506-900 Rio Claro - SP, Brasil \\ Paulo Sergio Tonello \\ Instituto de Química, Universidade Estadual Paulista, R. Francisco Degni, s/n, 14800-900 Araraquara - SP, Brasil \\ Rogério Caldorin \\ Instituto de Química de São Carlos, Universidade de São Paulo, CP 780, 13560-970 São Carlos - SP, Brasil
}

Recebido em 7/12/05; aceito em 16/5/06; publicado na web em 26/9/06

\begin{abstract}
PRECONCENTRATION OF CADMIUM WITH Saccharomyces cerevisiae AND DETERMINATION IN RIVER WATER USING INDUCTIVELY COUPLED PLASMA OPTICAL EMISSION SPECTROMETRY. A preconcentration method based on the use of Saccharomyces cerevisiae as sorbent material is proposed for the determination of $\mathrm{Cd}(\mathrm{II})$ in river water. The solid phase extraction was performed in batch mode and the determination of the analyte in the solid phase was easily carried out by introducing a slurry of the yeast $(0.0625 \mathrm{~g} / 2.5 \mathrm{~mL})$ directly into the ICP OES. A limit of detection of $0.11 \mu \mathrm{g} \mathrm{L}{ }^{-1}$ and a sample throughput in the range of 4 54 sample $h^{-1}$ were obtained. Determinations of cadmium in a certified sample and in real river water samples were in excellent agreement with the expected values.
\end{abstract}

Keywords: solid phase extraction; cadmium; yeast.

\section{INTRODUÇÃO}

O Cd é um metal extremamente tóxico, apresentando riscos à saúde quando consumido em concentrações superiores a $5 \mu \mathrm{g} \mathrm{L}^{-1}$ e visto como um dos principais poluentes para o mundo ${ }^{1}$. O metal pode contaminar sistemas aquáticos através da erosão do solo, de descargas na atmosfera devidas a operações industriais, vazamentos de aterros, pelo uso de lodos de esgoto e fertilizantes na agricultura. Também, podem ocorrer contaminações através de lançamento de efluentes industriais. Como consequiência da contaminação dos rios, os solos próximos podem ser atingidos pelo $\mathrm{Cd}$ através de irrigação para propósitos agrícolas, lançamentos de sedimentos dragados ou inundações ${ }^{1}$.

Os níveis de Cd para água doce, de chuva e de superfícies em áreas urbanas e industriais variam de $10 \mathrm{a} 4000 \mu \mathrm{g} \mathrm{L}^{-1}$, dependendo da região e de como o Cd é determinado (dissolvido ou total). Em águas superficiais ou subterrâneas não contaminadas ${ }^{1}$, usualmente, encontra-se em concentração menor que $1 \mu \mathrm{g} \mathrm{L^{-1 }}$.

Geralmente, a determinação de $\mathrm{Cd}$ em águas de superfícies ou subterrâneas não contaminadas requer a inclusão de uma etapa de pré-concentração do analito na análise, ainda que estejam disponíveis técnicas sensíveis, como a espectrometria de emissão óptica com plasma indutivamente acoplado (ICP OES), espectrometria de absorção atômica com chama (FAAS) e com forno de grafite (GFAAS).

Métodos de pré-concentração baseados na extração em fase sólida são considerados uma opção atrativa, em parte, por minimizarem a exposição a solventes, permitirem fatores de enriquecimento efetivos e robustez satisfatória para análise de vários tipos de amostras. Para determinação de Cd, são encontrados na literatura métodos baseados na retenção de $\mathrm{Cd}(\mathrm{II})$ em sorventes inorgânicos ${ }^{2}$, resinas adsortivas ${ }^{3,4}$, quelantes ${ }^{5-9}$ e catiônicas ${ }^{10}$. Uma outra abordagem proposta é fundamentada na retenção do complexo $\mathrm{CdCl}_{4}{ }^{2-} \mathrm{em}$ resina aniônica, após reação do metal com cloreto $^{11}$. Em uma revisão

*e-mail: amenega@rc.unesp.br relativamente recente, Camel $^{12}$ discute extensivamente as principais variantes do processo de extração em fase sólida, assim como algumas vantagens e limitações dos materiais sorventes utilizados para determinação de $\mathrm{Cd}$.

Também foram propostos como sorventes para separação e préconcentração de $\mathrm{Cd}$ alguns materiais alternativos, dentre os quais podem-se incluir, substâncias húmicas ${ }^{13}$ e microrganismos, como algas $^{14-16}$, bactérias ${ }^{17,18}$ e fungos ${ }^{19-21}$. Nestes trabalhos, as extrações foram conduzidas em batelada ou usando colunas contendo os substratos biológicos imobilizados. Por ex., o microrganismo Saccharomyces cerevisiae imobilizado em sepiolita e CPG ("Controlled Pore Glass") foi utilizado, respectivamente, por Bag et al. ${ }^{20}$ e Maquieira et al. ${ }^{21}$ para determinação de $\mathrm{Cd}$ em amostras de águas e digeridos de esgoto doméstico usando FAAS. O método proposto por Maquieira et al. ${ }^{21}$ foi caracterizado por um limite de detecção (LD) de $0,2 \mu \mathrm{g} \mathrm{L}^{-1}(2 \sigma)$ e uma frequiência analítica de 24 amostras $\mathrm{h}^{-1}$, enquanto Bag et al. ${ }^{20}$ relataram um LD de $44 \mu \mathrm{g} \mathrm{L}^{-1}(3 \sigma)$ e um tempo de 60 min para conduzir o processo de separação (incluindo as etapas de condicionamento da coluna, introdução da amostra e eluição).

Dentre os principais requerimentos práticos exigidos para um sorvente sólido ${ }^{12}$, pode-se considerar que a Saccharomyces cerevisiae é um material barato, acessível, apresenta alta capacidade de sorção ${ }^{20,21}$ e sorção quantitativa e rápida ${ }^{20,21}$. Também, em comparação a outros substratos biológicos, apresenta outras vantagens, como ser facilmente obtida na forma desidratada em grandes quantidades e não ser um microrganismo patogênico.

Uma outra característica interessante decorrente do uso de substratos biológicos como materiais sorventes em extrações em batelada é a possibilidade da determinação do analito em uma suspensão da fase sólida, pois, deste modo, eliminam-se as etapas de condicionamento do material e de eluição do analito, simplificando e agilizando o processo de separação. Esta abordagem foi utilizada com sucesso para determinação de As por geração de hidretos ${ }^{22}$, Ni, Co e $\mathrm{Cu}$ por $\mathrm{GFAAS}^{23,24}$, porém, até o momento, não foi relatada para determinação de $\mathrm{Cd}$ por 
ICP OES. Por outro lado, há uma certa relutância em utilizar substratos biológicos como material sorvente em química analítica, possivelmente, decorrente do fato de que microrganismos de diferentes linhagens podem ter comportamentos diferentes, dificultando assim especificar ou caracterizar o material utilizado no método analítico.

Neste trabalho avaliou-se o emprego de Saccharomyces cerevisiae de diversas procedências como material sorvente para pré-concentração de $\mathrm{Cd}$. Um método, baseado na extração em fase sólida utilizando este material é proposto para determinação de $\mathrm{Cd}$ em águas fluviais. $\mathrm{Na}$ abordagem proposta a extração é realizada em batelada e a fase sólida contendo o analito, depois de separada, é re-suspendida e nebulizada diretamente para o ICP OES.

\section{PARTE EXPERIMENTAL}

\section{Equipamentos e acessórios}

Foi utilizado um espectrômetro de emissão óptica sequiencial com plasma indutivamente acoplado marca GBC, modelo integra XL (Melbourne, Austrália) equipado com câmara de nebulização do tipo ciclônica (Glass Expansion, Melbourne, Austrália) e nebulizador V-Groove (Glass Expansion). Todas as determinações foram realizadas utilizando as seguintes condições de operação: potência incidente de $1200 \mathrm{~W}$; vazão do gás plasma, auxiliar e nebulização de $10,0,5$ e $0,6 \mathrm{~L} \mathrm{~min}^{-1}$, respectivamente; linha de emissão e altura de observação de 228,802 nm e 10 mm, respectivamente; vazão de introdução da amostra de $2,8 \mathrm{~mL} \mathrm{~min}^{-1}$.

Outros equipamentos utilizados foram um bloco digestor marca Tecnal modelo Te 40/25 (Piracicaba, São Paulo, Brasil) uma incubadora com agitação orbital marca Marconi modelo MA 420 (Piracicaba, São Paulo, Brasil) e uma centrífuga marca Jouan modelo B4i (St. Herblain, França).

\section{Reagentes e soluções}

Foram utilizadas leveduras desidratadas de três procedências distintas: uma obtida de uma usina sucro-alcooleira (linhagem ATCCFL97G3); duas obtidas no comércio local e destinadas à fabricação de pães (Comercial 1 e Comercial 2); uma destinada a uso laboratorial produzida pela Aldrich Chemical Company (Milwaukee, EUA).

O ácido nítrico utilizado foi grau p.a. (Merck, Darmstadt) e todas as soluções foram preparadas usando água purificada a uma resistividade de $18 \mathrm{M} \Omega \mathrm{cm}$ (Milli-Q, Millipore, Bedford, EUA).

Foram utilizadas soluções padrão de 0 - $1000 \mu \mathrm{g} \mathrm{L} \mathrm{L}^{-1} \mathrm{de} \mathrm{Cd}(\mathrm{II})$ preparadas a partir de diluições sucessivas de uma solução estoque $1000 \mathrm{mg} \mathrm{L}^{-1}$ (High-Purity Standards, Charleston, EUA).

Soluções de $\mathrm{Fe}(\mathrm{III}), \mathrm{Cu}(\mathrm{II}), \mathrm{Zn}(\mathrm{II}), \mathrm{Ca}(\mathrm{II}), \mathrm{Mg}(\mathrm{II}), \mathrm{K}(\mathrm{I})$ e $\mathrm{Na}(\mathrm{I})$, utilizadas no teste de interferências, foram preparadas a partir de $\mathrm{FeCl}_{3} \cdot 6 \mathrm{H}_{2} \mathrm{O}$ (Synth, São Paulo, Brasil), $\mathrm{CuSO}_{4}$ (Mallinckrodt, EUA), $\mathrm{ZnSO}_{4}$ (Merck), $\mathrm{CaCO}_{3}$ (Merck), $\mathrm{MgCl}_{2} \cdot 6 \mathrm{H}_{2} \mathrm{O}$ (Merck), $\mathrm{KCl}$ (Synth) e $\mathrm{NaCl}$ (Synth), respectivamente.

\section{Procedimentos de extração em fase sólida e pré-concentração}

O procedimento geral da extração em fase sólida constituiu em se adicionar uma alíquota $(10-40 \mathrm{~mL})$ da solução em teste em um tubo de centrífuga $(15-50 \mathrm{~mL})$ contendo uma quantidade estabelecida do substrato (Saccharomyces cerevisiae), incubar a mistura e, posteriormente, separar as fases sólida e líquida por centrifugação durante 5 min a $4200 \mathrm{rpm}$. Em todos os experimentos o $\mathrm{pH}$ das soluções foi ajustado em 7,0 $\pm 0,1$, usando uma solução de $\mathrm{NaOH} 0,1 \mathrm{~mol} \mathrm{~L}^{-1}$. Foi realizada uma homogeneização prévia da solução em teste com a levedura antes da etapa de incubação, agi- tando os tubos manualmente durante $30 \mathrm{~s}$.

Nos testes preliminares, visando avaliar as leveduras de diferentes procedências, a determinação de Cd na fase sólida foi realizada após a decomposição ácida do material, segundo procedimento descrito a seguir. Nos experimentos, tendo como principal objetivo a pré-concentração do analito, a fase sólida foi retomada com $2,5 \mathrm{~mL}$ de $\mathrm{HNO}_{3} 2 \%$ (v/v) e a suspensão nebulizada diretamente para o ICP.

\section{Procedimento de decomposição da fase sólida (levedura)}

Depois da etapa de extração, a biomassa foi pré-digerida adicionando-se $5 \mathrm{~mL}$ de $\mathrm{HNO}_{3}$ concentrado aos frascos e mantendo-os em temperatura ambiente durante $24 \mathrm{~h}$. Posteriormente, as misturas foram quantitativamente transferidas para tubos de digestão e aquecidas a $100{ }^{\circ} \mathrm{C}$ por $30 \mathrm{~min}$ e, em seguida, a $150{ }^{\circ} \mathrm{C}$ por $3 \mathrm{~h} . \mathrm{O}$ resíduo foi avolumado com água a um volume final de $40 \mathrm{~mL}$.

\section{Preparo das amostras de águas fluviais}

Foram analisadas cinco amostras de águas de rios, sendo uma certificada pelo "National Institute of Standard and Technology" (NIST "Standard Reference Material" 1640). Depois de coletadas, as amostras foram filtradas em membrana de microfibra de vidro $(1,2 \mu \mathrm{m})$.

$\mathrm{O}$ preparo das amostras de água consistiu no ajuste do $\mathrm{pH}$ entre 6,5 a 7,5. Logo depois, foram tomadas três alíquotas de $40 \mathrm{~mL}$ para os frascos de centrífuga contendo $0,0625 \mathrm{~g}$ de biomassa e realizada a extração do analito.

\section{RESULTADOS E DISCUSSÃO}

\section{Procedência do substrato}

Inicialmente, foram realizados experimentos visando avaliar possíveis alterações na retenção de $\mathrm{Cd}(\mathrm{II})$ decorrentes da variabilidade de linhagens da Saccharomyces cerevisiae. Deste modo, realizaram-se extrações usando $0,500 \mathrm{~g}$ das diferentes leveduras e alíquotas de $40 \mathrm{~mL}$ de uma solução padrão de $\mathrm{Cd}(\mathrm{II}) 200 \mu \mathrm{g} \mathrm{L} \mathrm{L}^{-1}$. Depois de agitadas manualmente, as suspensões foram incubadas em um banho de água a $25^{\circ} \mathrm{C}$ durante $60 \mathrm{~min}$.

Para as quatro leveduras avaliadas, foram obtidas retenções de Cd(II) (média \pm limite de confiança, nível de $95 \%, \mathrm{~N}=3$ ) de $81 \pm 9$, $84 \pm 2,86 \pm 15,88 \pm 4 \%$, mostrando que não houve diferença significativa na retenção do analito por leveduras de procedências distintas. Também, não se verificou contaminação de $\mathrm{Cd}$ em nenhuma das leveduras avaliadas, apesar de quantidades consideráveis de $\mathrm{Cu}$ e $\mathrm{Zn}$ serem encontradas nas provas em branco dos quatro substratos (Figura 1). Os experimentos subseqüentes foram realizados utilizando a levedura adquirida da Aldrich.

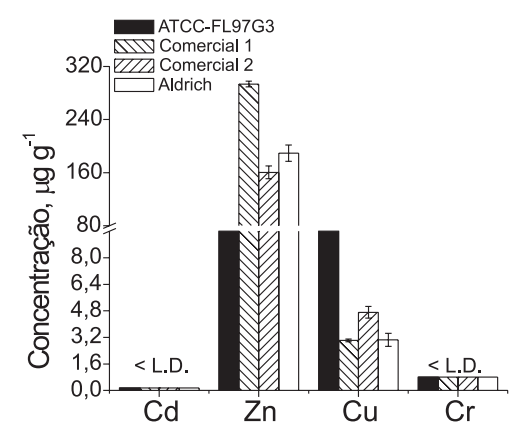

Figura 1. Concentração de $\mathrm{Cd}, \mathrm{Cr}$, Cu e Zn encontradas na prova em branco da fase sólida (Saccharomyces cerevisiae de diversas procedências). Os valores atribuídos ao Cd e Cr referem-se aos limites de detecção (L. D.) 


\section{Determinação de Cd na fase sólida através da nebulização direta da suspensão de levedura}

Visando eliminar a etapa de eluição (ou digestão) do processo de extração, avaliou-se a possibilidade de determinar o analito na fase sólida, nebulizando suspensões da levedura diretamente para o ICP. Para isto, realizaram-se testes de recuperação adicionando o analito em suspensões contendo quantidades crescentes da levedura.

As recuperações de $\mathrm{Cd}$ em suspensões de 0,250, 0,625, 1,25, 2,50 e $6,25 \%$ (g por $100 \mathrm{~mL}$ de $\mathrm{HNO}_{3} 2 \%$, v/v), quantificadas usando soluções padrão em $\mathrm{HNO}_{3} 2 \%$ (v/v), foram $87 \pm 9,90 \pm 13,87 \pm$ $3,86 \pm 1$ e $84 \pm 5 \%$, respectivamente. Estes resultados mostram que o efeito causado pela matriz foi caracterizado por uma pequena supressão do sinal do analito (entre 10 a 16\%), evidenciando a viabilidade de descartar a etapa de eluição do processo de extração.

\section{Pré-concentração}

Visando otimizar a pré-concentração de Cd(II) foram testados procedimentos de incubação com e sem agitação.

O procedimento sem agitação consistiu em incubar a mistura em um banho de água a $25^{\circ} \mathrm{C}$ por 10 min enquanto que, no procedimento com agitação, os tubos contendo a mistura foram colocados em uma incubadora orbital com controle de temperatura e agitados durante 10 min a $25{ }^{\circ} \mathrm{C}$. Os dois procedimentos de incubação foram avaliados usando 0,0625 g de levedura e alíquotas de 10, 25 e 40 $\mathrm{mL}$ de uma solução padrão $10 \mu \mathrm{g} \mathrm{L^{-1 }}$ de Cd(II). Depois de separada, a fase sólida foi retomada com 2,5 mL de $\mathrm{HNO}_{3} 2 \%(\mathrm{v} / \mathrm{v})$ e a suspensão nebulizada diretamente para o ICP.

Na Tabela 1 são apresentados os fatores de pré-concentração obtidos com e sem agitação. Estes foram calculados dividindo-se a sensibilidade obtida com a nebulização da suspensão da fase sólida (após a etapa de extração) pela sensibilidade obtida com a nebulização de soluções padrão de $\mathrm{Cd}$ em $\mathrm{HNO}_{3} 2 \%$ (v/v). Os valores apresentados na Tabela 1 mostram que o procedimento de pré-concentração utilizado permitiu um aumento de sensibilidade de até 14 vezes, quando comparado com o procedimento de análise convencional.

Tabela 1. Fatores de pré-concentração para $\mathrm{Cd}(\mathrm{II})$, média $\pm \mathrm{sd}$, obtidos usando diferentes volumes de solução padrão. $\mathrm{N}=3$

\begin{tabular}{lcc}
\hline Volume, $\mathrm{mL}$ & Com agitação & Sem agitação \\
\hline 10 & $3,4 \pm 0,1$ & $3,3 \pm 0,2$ \\
25 & $8,9 \pm 0,2$ & $7,3 \pm 0,2$ \\
40 & $14 \pm 1$ & $10 \pm 1$ \\
\hline
\end{tabular}

Quando um volume de $10 \mathrm{~mL}$ foi utilizado, o emprego da agitação no processo de extração pouco afetou o fator de pré-concentração. Por outro lado, a inclusão de agitação no processo de extração resultou em aumentos significativos dos fatores de pré-concentração para volumes superiores a $10 \mathrm{~mL}$. Na Figura 2 observase claramente que a agitação resulta em um aumento na taxa de retenção do $\mathrm{Cd}(\mathrm{II})$ em extrações utilizando volumes maiores que $10 \mathrm{~mL}$ e que, em todos os casos, o íon foi removido quantitativamente da fase líquida. Também, as repetibilidades das extrações foram melhores quando a agitação foi incluída no processo.

Um teste adicional mostrou que $0,0625 \mathrm{~g}$ do substrato foi suficiente para reter $10 \mu \mathrm{g}$ de $\mathrm{Cd}(\mathrm{II})$. Isto representa uma capacidade de retenção de $\mathrm{Cd}(\mathrm{II})$ pela levedura de, pelo menos, 1,42 $\mu \mathrm{mol}$ $\mathrm{Cd}(\mathrm{II}) / \mathrm{g}$ de substrato.

Baseando-se nestes resultados, os testes subseqüentes foram realizados utilizando $0,0625 \mathrm{~g}$ de levedura e agitação no processo de extração.

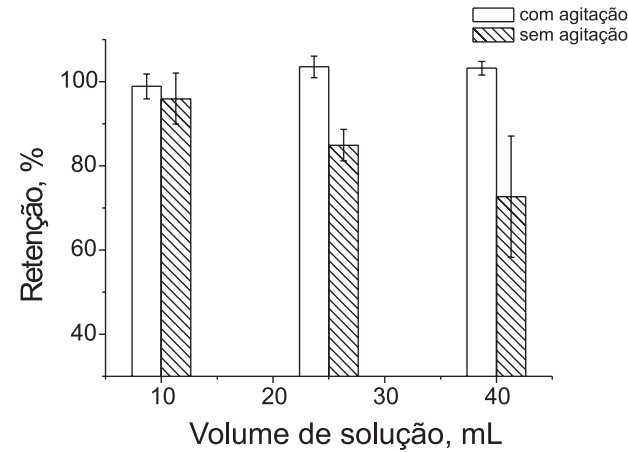

Figura 2. Retenção de Cd(II) pela levedura (Aldrich) usando extrações em fase sólida em batelada com e sem agitação. Temperatura $=25^{\circ} \mathrm{C}$; biomassa $=0,0625 \mathrm{~g}$; tempo de incubação $=10 \mathrm{~min}$

\section{Interferências}

O teste de interferência foi conduzido usando alíquotas de 40 $\mathrm{mL}$ de soluções contendo $10 \mu \mathrm{g} \mathrm{L}^{-1}$ de $\mathrm{Cd}(\mathrm{II})$ e o potencial interferente. Foram avaliadas possíveis interferências decorrentes da presença de $\mathrm{Fe}(\mathrm{III}), \mathrm{Cu}(\mathrm{II}), \mathrm{Zn}(\mathrm{II}), \mathrm{Ca}(\mathrm{II}), \mathrm{Mg}(\mathrm{II}), \mathrm{K}(\mathrm{I})$ ou $\mathrm{Na}(\mathrm{I})$ em razões de concentrações potencial interferente:analito de 100, 100, 100, 400, 400, 800 e 800, respectivamente.

As recuperações de $\mathrm{Cd}(\mathrm{II})$ (média $\pm \mathrm{sd}, \mathrm{N}=3$ ) na fase sólida para soluções contendo o analito e $\mathrm{Fe}(\mathrm{III}), \mathrm{Cu}$ (II), $\mathrm{Zn}$ (II), $\mathrm{Ca}$ (II), $\mathrm{Mg}(\mathrm{II}), \mathrm{K}(\mathrm{I})$ ou $\mathrm{Na}$ (I) foram $97 \pm 2,100 \pm 5,98 \pm 1,97 \pm 1,101 \pm 1$, $96 \pm 1,98 \pm 1 \%$, respectivamente. Nenhum dos íons afetou significativamente a retenção de $\mathrm{Cd}(\mathrm{II})$, de acordo com o teste $t(\mathrm{~N}=3$, nível de confiança de 99\%), indicando que o procedimento desenvolvido é suficientemente robusto para análise de amostras reais.

\section{Determinação de Cd(II) em amostras de águas fluviais}

Para determinação de $\mathrm{Cd}$ (II) nas amostras de águas fluviais, além das amostras, um conjunto de soluções padrão (entre 0,0 - 10,0 $\mu \mathrm{g} \mathrm{L}^{-1}$ ) em $\mathrm{pH}=7,0 \pm 0,1$ também foi submetido ao procedimento de extração. Uma curva de calibração construída com os sinais provenientes da nebulização da suspensão do respectivo conjunto foi utilizada para determinação de $\mathrm{Cd}(\mathrm{II})$ nas amostras. Ressalta-se que esta estratégia de quantificação do $\mathrm{Cd}$ na fase sólida não requer o emprego de técnicas de padronização para compensar os efeitos interferentes decorrentes da nebulização da suspensão, pois amostras e padrões apresentam a mesma quantidade de substrato.

Foi obtida uma curva de calibração linear $(y=1310 x+326)$ caracterizada por um $\mathrm{R}^{2}$ de 0,999 , sendo "y" a intensidade do sinal e "x" a concentração em $\mu \mathrm{g} \mathrm{L}{ }^{-1}$. O limite de detecção do Cd, considerando um critério $3 \sigma$, foi $0,1 \mu \mathrm{g} \mathrm{L}^{-1}$. O desvio padrão relativo das medidas dos sinais $(\mathrm{N}=12)$ obtido para uma solução contendo 10 $\mu \mathrm{g} \mathrm{\textrm {L } ^ { - 1 }}$ de $\mathrm{Cd}(\mathrm{II})$ foi $1,7 \%$.

Considerando um limite mínimo de 1 e um máximo de 16 (limitação imposta pelo rotor da centrífuga) para o número de amostras que podem ser processadas simultaneamente e, também, incluindo um tempo de leitura no ICP OES de $10 \mathrm{~s}$ (para cada amostra), podese estimar uma frequiência analítica (considerando apenas o tempo de instrumentação) para o método proposto entre 4 a 54 amostras $\mathrm{h}^{-1}$.

Na Tabela 2 são apresentados os resultados das análises das amostras de águas fluviais. Mesmo para amostras contendo concentrações de Cd inferiores a $1 \mu \mathrm{g} \mathrm{L}^{-1}$ foi possível a determinação do analito, mostrando a aplicabilidade do método para análise de águas fluviais não contaminadas. Para todas as amostras não certificadas foram obtidas recuperações quantitativas de $\mathrm{Cd}(\mathrm{II})$ (entre $94 \mathrm{e}$ $105 \%$ ) e a concentração de Cd obtida para o material de referência 
(NIST - SRM 1640) foi concordante com o valor certificado, demonstrando que a exatidão do método desenvolvido foi satisfatória.

Tabela 2. Determinação de Cd (média \pm sd) em amostras de águas fluviais. $\mathrm{N}=3$

\begin{tabular}{lcc}
\hline Amostra & Encontrado, $\mu \mathrm{g} \mathrm{L}^{-1}$ & Recuperação \\
\hline 1 R1 & $0,30 \pm 0,05$ & $95 \pm 1^{\mathrm{a}}$ \\
2 R2 & $0,18 \pm 0,05$ & $99 \pm 2^{\mathrm{a}}$ \\
3 R3 & $0,16 \pm 0,02$ & $94 \pm 5^{\mathrm{a}}$ \\
1 FHD & $2,0 \pm 0,1$ & $105 \pm 2^{\mathrm{a}}$ \\
NIST - SRM 1640 & $21,0 \pm 1,5$ & $92 \pm 7^{\mathrm{b}}$ \\
\hline
\end{tabular}

${ }^{a}$ referente a adições de $100 \mathrm{ng}$ de $\mathrm{Cd}(\mathrm{II})$; ${ }^{\mathrm{b}}$ referente ao valor certificado $\left(22,79 \pm 0,96 \mu \mathrm{g} \mathrm{L}^{-1}\right)$.

\section{CONCLUSÕES}

Os estudos realizados mostraram a viabilidade de se usar a Saccharomyces cerevisiae como material sorvente para pré-concentrar $\mathrm{Cd}$, através de extrações em fase sólida em batelada. Esta propriedade, combinada com a possibilidade de nebulizar diretamente a suspensão do material sorvente para o ICP, foi utilizada com sucesso para desenvolver um método sensível e robusto para determinação de Cd.

O procedimento de extração adotado é simples, barato e rápido, principalmente considerando-se a parte instrumental do processo, em parte, porque a nebulização direta da suspensão da fase sólida dispensou as etapas de condicionamento do material sorvente e a eluição do analito. O consumo da levedura não representa um problema porque o material pode ser obtido em grandes quantidades e a preços módicos.

Não são esperados erros decorrentes de variabilidade de linhagens da Saccharomyces cerevisiae e, mesmo que ocorram, podem ser minimizados com a estratégia de quantificação utilizada.

$\mathrm{O}$ método proposto foi efetivo para determinação de $\mathrm{Cd}$ em amostras de águas fluviais, apresentando características analíticas (limite de detecção e freqüência analítica) comparáveis ou superiores a métodos baseados em extrações com colunas usando o microrganismo imobilizado.

\section{AGRADECIMENTOS}

À Fundação de Amparo à Pesquisa do Estado de São Paulo (FAPESP) pelo apoio financeiro.

\section{REFERÊNCIAS}

1. Chasin, A. A. M.; Cardoso, L. M. N. Em Metais: Gerenciamento da Toxidade; de Azevedo, F. A.; Chasin, A. A. M., eds.; Atheneu: São Paulo, 2003, cap. 10 .

2. Vassileva, E.; Proinova, I.; Hadjiivanov, K.; Analyst 1996, 121, 607.

3. Abollino, O.; Mentasti, E.; Porta, V.; Sarzanini, C.; Anal. Chem. 1990, 62, 21

4. Saraçoglu, S.; Elçi, L.; Anal. Chim. Acta 2002, 452, 77.

5. Saxena, R.; Singh, A. K.; Anal. Chim. Acta 1997, 340, 285.

6. Saxena, R.; Singh, A. K.; Sambi, S. S.; Anal. Chim. Acta 1994, 295, 199.

7. Tewari, P. K.; Singh, A. K.; Analyst 2000, 125, 2350.

8. Ramesh, A.; Mohan, K. R.; Seshaiah, K.; Talanta 2002, 57, 243.

9. Pai, S. C.; Whung, P. Y.; Lai, R. L.; Anal. Chim. Acta 1988, 211, 257.

10. Menegário, A. A.; Giné, M. F.; Spectrochim. Acta, Part B 2001, 56, 1917.

11. Gomes Neto, J. A.; Oliveira, A. P.; Feshi, G. P. G.; Dakuzaku, C. S.; Moraes, M.; Talanta 2000, 53, 497.

12. Camel, V.; Spectrochim. Acta, Part B 2003, 58, 1177.

13. Pereira, M. D.; Arruda, M. A. Z; Mikrochim. Acta 2004, 146, 215.

14. Majidi, V.; Holcombe, J. A.; Spectrochim. Acta, Part B 1988, 43, 1423.

15. Majidi, V.; Holcombe, J. A.; J. Anal. At. Spectrom. 1989, 4, 439.

16. Mahan, C. A.; Majidi, V.; Holcombe, J. A.; Anal. Chem. 1989, 61, 624.

17. Bag, H.; Turker, A. R.; Lale, M.; Talanta 2000, 51, 1035.

18. Robles, L. C.; Aller, A. J.; Quim. Anal. 1996, 15, 21.

19. Bag, H.; Turker, A. R.; Lale, M.; Anal. Sci. 1999, 15, 1251.

20. Bag, H.; Lale, M.; Turker, A. R.; Fresenius J. Anal. Chem. 1999, 363, 224.

21. Maquieira, A.; Elmahadi, H. A. M.; Puchades, R.; Anal. Chem. 1994, 66 , 1462.

22. Smichowski, P.; Marrero, J.; Ledesma, A.; Polla, G.; Batistoni, D. A.; J. Anal. At. Spectrom. 2000, 15, 1493.

23. Shengjun, M.; Holcombe, J. A.; Talanta 1991, 38, 5.

24. Mo, S. J.; Holcombe, J. A.; Anal. Chem. 1990, 62, 1994. 